\title{
Relative quantification of Porphyromonas gingivalis, Treponema denticola, Tannerella forsythia and Aggregatibacter actinomycetemcomitans high-risk bacterial species in
}

\section{Romanian patients evaluated for periodontal disease}

\author{
Cosac Ion Constantin ${ }^{1}$, Ionica Consuel ${ }^{1,2}$, Ratiu Attila Cristian ${ }^{1,3}$, Savu Lorand $^{1,4}$ \\ ${ }^{1}$ GeneticLab, Capitan Aviator Nicolae Drossu Street No. 9, Bucharest, Romania \\ 2 “F. I. Rainer" Anthropology Institute, Casa Academiei, 13 Septembrie No. 13 Ave., Bucharest, Romania \\ ${ }^{3}$ Department of Genetics, Faculty of Biology, University of Bucharest, Intrarea Portocalelor Street, No. 1-3, Bucharest, Romania \\ ${ }^{4}$ Faculty of Medicine, Titu Maiorescu University, Gheorghe Petraşcu No. 67A Street, Bucharest, Romania
}

\begin{abstract}
Three bacterial species pertaining to the red complex (Porphyromonas gingivalis, Treponema denticola, and Tannerella forsythia) and Aggregatibacter actinomycetemcomitans were investigated in relation to the incidence and severity of periodontal disease. A total of 259 patients were included in this study, 179 being diagnosed with periodontal disease. The gingival crevicular fluid samples were obtained from periodontal pockets and the presence and levels of target bacteria were assessed following DNA extraction and real-time quantitative PCR. Our results account for significant positive associations between the number of bacterial species from the red complex coexisting within a patient and several clinical signs (gingival bleeding, inflammation and bone deterioration). A similar positive association was found between bacterial load of the red complex species and the clinical Case Type diagnostic of the periodontal disease, as well as the probing depth with the most evident results for T. denticola. In conclusion, our study, a first for the Romanian population, confirms previous results found elsewhere and finds a possible regional pathogenic specificity for $T$. denticola as a major factor for periodontitis severity.
\end{abstract}

Key words: periodontal disease, bacterial load, Porphyromonas gingivalis, Treponema denticola, Tannerella forsythia, Aggregatibacter actinomycetemcomitans

\section{Introduction}

Periodontal disease is a multifactorial affliction that is becoming increasingly common in middle-aged people, mainly due to the lack of attention to distinctive clinical signs (Chen et al, 2005). Genetic predisposition, lifestyle (smoking, stress, and oral hygiene), as well as socio-economic status, precede and modulate the development of the bacterial biofilm present in the gingival sulcus, which represents the main cause of gingivitis and periodontal disease (Mariotti and Arthur, 2015; Chen et al, 2005).

Periodontal disease is commonly recognized as the association between gingival inflammation and the pathological detachment of the connective tissue (Armitage, 1995). Gingival inflammation is mainly caused by infections with specific groups of oral bacteria 
(Armitage, 2003). The treatment of periodontal disease consists in both surgical (gum surgery and bone grafts) and non-surgical approaches (removal of the microbial plaque and administration of antibiotics). Identification of pathogenic species of bacteria is essential for the antibiotic treatment (Loloya-Rodriguez et al., 2014). In addition, quantifying the bacterial load can help monitoring the progression of the treatment.

Several methodologies were used to investigate the periodontal pathogenic bacterial load for therapy purposes, such as targeting 16S RNA genes (Armitage, 2003). According to the previous clinical assessments, periodontal pathogenic bacteria are usually grouped into three main classes (or complexes): red, orange and green. The Gram-negative anaerobic bacteria in the red complex (P. gingivalis, T. denticola, T. forsythia), representing the most aggressive class, along with $A$. actinomycetemcomitans from the green complex, are believed to be major etiologic agents for determining the severity of periodontal disease (Decat et al., 2012; The Research, Science and Therapy Committee of the American Academy of Periodontology, 1999; Tettamanti et al., 2017). Bacteria complexes, other than the red one, are also important but have a lesser impact on the severity of the disease. It appears that orange and green complexes are associated with long term gingivitis, which precedes the actual periodontal disease, while the red complex are the late colonizers of the plaque biofilm (Teles et al., 2013). A. actinomycetemcomitans has been found to be one of the first and most important etiological agents that invades the subgingival plaque and set up the environment for other bacterial species (Mariotti and Arthur, 2015).

Several studies regarding the prevalence and bacterial load of various species of pathogenic bacteria found differences among the focus populations (Tettamanti et al., 2017). These results are relevant in order to successfully elaborate strategies using specific antibiotics for targeting the most frequent/aggressive bacterial strains in a variety of populations.

Our study represents the first assessment of the pathogenic bacteria responsible for periodontal diseases in Romanian patients, its main objective being to assess the correspondence between the presence and load of the red complex bacteria and respectively A. actinomycetemcomitans with various levels of periodontal disease.

\section{Materials and methods}

Subject population - This study included 259 periodontally untreated patients with an average age of 44 years $(\mathrm{sd} \pm 11), 51 \%$ of them being women. Additional information on evaluated patients included self-recorded categories of age, sex, and smoker status, as well as 
rarely stated pregnancy and relevant medical conditions such as diabetes, immunodepression, heart diseases or hereditary periodontal disease. Supplementary data provided by the dental clinic included description of symptoms (gingival bleeding, redness, inflammation, bone deterioration, and halitosis), clinical degree of periodontal disease (Case Type I - gingivitis, Case Type II - mild periodontitis, Case Type III - moderate periodontitis, Case Type IV advanced periodontitis, and Case Type V - refractory periodontitis) (Armitage, 2003), and depth of pocket probing (range of $1 \mathrm{~mm}$ to $15 \mathrm{~mm}$ ).

Sample collection - Samples of gingival crevicular fluid were collected at the dental clinic using five sterile paper tips, each inserted into a different periodontal pocket and held there for approximately 10 seconds. Afterwards, the paper tips were transferred to a single sterile tube and sent to our laboratory for DNA extraction and bacterial quantification. Therefore, total bacterial load represented a pool of different species present in the tested periodontal pockets.

Bacterial DNA extraction - Paper tips were resuspended overnight in $1 \mathrm{~mL}$ of $1.5 \mathrm{X}$ PBS solution. Bacterial pellets were obtained after 10 minutes of centrifugation at 16,000 $\mathrm{g}$ and, subsequently, $200 \mu \mathrm{L}$ of PBS containing the resuspended bacteria were further processed using the Viral Kit I (Favorgene) according to manufacturer's instructions. Briefly, samples were supplemented with $200 \mu \mathrm{L}$ of PBS and $570 \mu \mathrm{L}$ of VNE buffer (enriched with DNA carrier) and, after 10 minutes of incubation at room temperature, $570 \mu \mathrm{L}$ absolute ethanol were added. Subsequently, the resulting solutions were loaded on individual DNA separation columns that were washed one time with $500 \mu \mathrm{L}$ wash buffer I and two times with $750 \mu \mathrm{L}$ wash buffer II. Finally, DNA was eluted in $80 \mu \mathrm{L}$ pre-heated nuclease-free water.

Real-time quantitative PCR ( $q R T-P C R)$ - Bacterial quantification was performed on a LightCycler 2.0 machine (Roche). Single PCRs targeting individual bacterial species were performed in a total volume of $20 \mu \mathrm{L}$ containing 1.25X colourless buffer (Promega), 0.25 $\mathrm{mM}$ dNTPs, $4 \mathrm{mM} \mathrm{MgCl} 2,1.5$ units of PQ Taq (Promega), $1 \mu \mathrm{L}$ of 1 X SYBR Green I, 0.5 $\mathrm{mM}$ of each primer (primer sequences are listed in table 1) and $5 \mu \mathrm{L}$ of DNA (Kirakodu et al., 2008; Masunaga et al., 2010; Bastos et al.,2011).

The amplification comprised of an initial 5 minutes denaturation at $95^{\circ} \mathrm{C}, 40$ cycles consisting in 10 seconds at $95^{\circ} \mathrm{C}, 5$ seconds at $55^{\circ} \mathrm{C}$, and 25 seconds at $72^{\circ} \mathrm{C}$, followed by High Resolution Melt (HRM) analysis in order to monitor the species-specific melting point.

The relative quantitation of bacterial load was achieved by comparison with a standard amplification curve obtained starting from genomic DNA corresponding to 6.0E6 colony founding units (CFUs) of $P$. gingivalis isolated from pure cultures. 
The LightCycler 1.5 software was used to infer the cycle threshold $(\mathrm{Ct})$ values and to perform the HRM analysis.

Table 1. Primer sequences used for PCR amplifications.

\begin{tabular}{|c|c|c|c|c|}
\hline Bacterial species & Forward primer & Reverse primer & $\begin{array}{c}\text { Amplicon } \\
\text { length (bp) }\end{array}$ & $\begin{array}{c}\text { Melting } \\
\text { temperature ('⿳C㇒一 }\end{array}$ \\
\hline A. actinomycetemcomitans & 5' AAACCCATCTCTGAGTTCTTCTTC 3' & 5' ATGCCAACTTGACGTTAAAT 3' & 557 & 87 \\
\hline P. gingivalis & 5' AGGCAGCTTGCCATACTGCG 3' & 5' ACTGTTAGCAACTACCGATGT 3' & 404 & 87 \\
\hline T. denticola & 5' TAATACCGAATGTGCTCATTTACAT 3' & 5' TCAAAGAAGCATTCCCTCTTCTTCTTA 3' & 316 & 88 \\
\hline T. forsythia & 5' GCGTATGTAACCTGCCCGCA 3' & 5' TGCTTCAGTGTCAGTTATACCT 3' & 641 & 87 \\
\hline
\end{tabular}

Statistical analysis -We applied chi-square analysis to test for association between the number of red-complex species present per patient and presence of each symptom. To assess each species' load as a function of probing depth we employed Pearson correlation. The same test was used to evaluate the association of pairs of species. Finally, a Kruskal-Wallis nonparametric ANOVA was applied separately to each species' bacterial load to test for the association with the severity of periodontal disease as clinically diagnosed by dentists (i.e. Case Type). All tests were performed using the Minitab v.16 software or R software environment using a $5 \%$ level of significance, two tailed.

\section{Results and Discussions}

In our approach, each bacterial species was targeted by individual qRT-PCR reactions containing specific primer pairs. The LightCycler 1.5 software computed the $\mathrm{Ct}$ values that were compared with the $\mathrm{Ct}$ inferred from the amplification curve of a standard sample containing genomic DNA equivalent to 6E6 CFUs of $P$. gingivalis. The target amplicons corresponding to specific bacterial species have different lengths and this feature could impact the amplification efficiency, due to their increased size that enhances the assay sensitivity (Peirson et al., 2003). However, the fact that Ct values are estimated in the early cycles of exponential amplification, and considering that errors of up to few hundred percent may be tolerated when trends or relative big changes in amounts are measured (Bar et al., 2012), PCR efficiencies are adequately comparable for the study's purpose.

Bacterial load of all species ranged between 1.0E6 and 1.0E14 CFUs (table 2). Samples were positive for at least one ( $\mathrm{N}=10,3.86 \%$ of cases), two $(\mathrm{N}=42,16.22 \%$ of cases), three ( $\mathrm{N}=151,58.30 \%$ of cases $)$ and all four $(\mathrm{N}=49,18.92 \%$ of cases $)$ investigated pathogenic bacteria, with varying degrees of abundance across species. Several samples $(\mathrm{N}=$ 
$155,59.8 \%$ of cases) were also positive for additional bacteria pertaining to orange and green complexes (data not shown).

A. actinomycetemcomitans was by far the least present species $(\mathrm{N}=61,24 \%$ of cases), while the other bacterial species were detected more frequently: T. denticola $(\mathrm{N}=$ $219,85 \%$ of cases), $T$. forsythia $(\mathrm{N}=227,88 \%$ of cases $)$, and $P$. gingivalis $(\mathrm{N}=236,91 \%$ of cases).

Table 2. The values of bacterial load distribution among tested samples.

\begin{tabular}{|c|c|c|c|}
\hline Bacterial species & Mean CFUs & Standard deviation & Median CFUs \\
\hline A. actinomycetemcomitans & E9.87 & E1.98 & E10 \\
\hline P. gingivalis & E12.9 & E1.83 & E14 \\
\hline T. denticola & E12 & E1.96 & E12 \\
\hline T. forsythia & E12.6 & E1.71 & E13 \\
\hline
\end{tabular}

Student's t-test and regression analysis showed that there are no associations between bacterial loads and patients' gender or age, results consistent with those provided by other studies (Farias et al., 2012).

Evaluation of the bacterial community comprising the analysed species indicated significant positive association between T. denticola and T. forsythia $(\mathrm{r}=0.589, \mathrm{p}<0.001)$, P. gingivalis and T. denticola $(\mathrm{r}=0.437, \mathrm{p}<0.001)$, . gingivalis and T. forsythia $(\mathrm{r}=0.470$, $\mathrm{p}<0.001)$, and A. actinomycetemcomitans and T. forsythia $(\mathrm{r}=0.354, \mathrm{p}=0.006)$, when tested as bacterial load using Pearson correlation. In contrast, we did not find significant associations between $T$. denticola and A. actinomycetemcomitans $(\mathrm{r}=-0.058, \mathrm{p}=0.674)$, and respectively $P$. gingivalis and $A$. actinomycetemcomitans $(r=0.010, p=0.945)$. The latter situation, illustrating a seeming conflict between the involved bacterial strains, could be explained by the fact that the major virulence factors of $P$. gingivalis consisting in the extracellular proteinases called Lys-gingipains (Gorman et al., 2015) are involved in the detachment and decrease of A. actinomycetemcomitans biofilms (Haraguchi et al., 2014). The $P$. gingivalis gingipains seem to also have positive effects on $T$. forsythia growth and are crucial for its co-adhesion with T. denticola (Bao et al., 2014) thus explaining their strong association at the same lesion. A previous study investigating the grouping of bacterial species existing in subgingival plaque found that the most tightly related group, forming a major complex related to worsened pocket depth and gingival bleeding, was composed of $P$. gingivalis, T. denticola and T. forsythia (Socransky et al., 1998). Interestingly, the surprising significant association between A. actinomycetemcomitans and T. forsythia could be in fact the consequence of the fact that the latter uses other virulence mechanisms instead of Lys- 
gingipains (Friedrich et al., 2015), thus probably not hindering the development of $A$. actinomycetemcomitans.

We tested for associations between the presence or absence of various clinical symptoms scored for patients and the number of bacterial species pertaining to the red complex. Some of the cases exhibited more than one symptom, thus they were counted each time an individual symptom was considered. Chi-square analysis of data representing the number of cases without or with symptoms when none, one, two, or all three red complex species were present, did not yield significant trends when redness or halitosis were analysed, but showed strong statistical significance $\left(\mathrm{p}<10^{-3}\right)$ for gingival bleeding, inflammation and bone deterioration. These symptoms were exceptionally common in cases displaying three bacterial species, more precisely, 167, 124 and 147 patients presenting gingival bleeding, inflammation or bone deterioration, respectively (figure 1). Similar results were previously reported when coexistence of all three species from the red complex determined increases in maxillary and mandibular alveolar bone resorption (Suzuki et al., 2013) or periodontal disease severity (Lanza et al., 2016). 


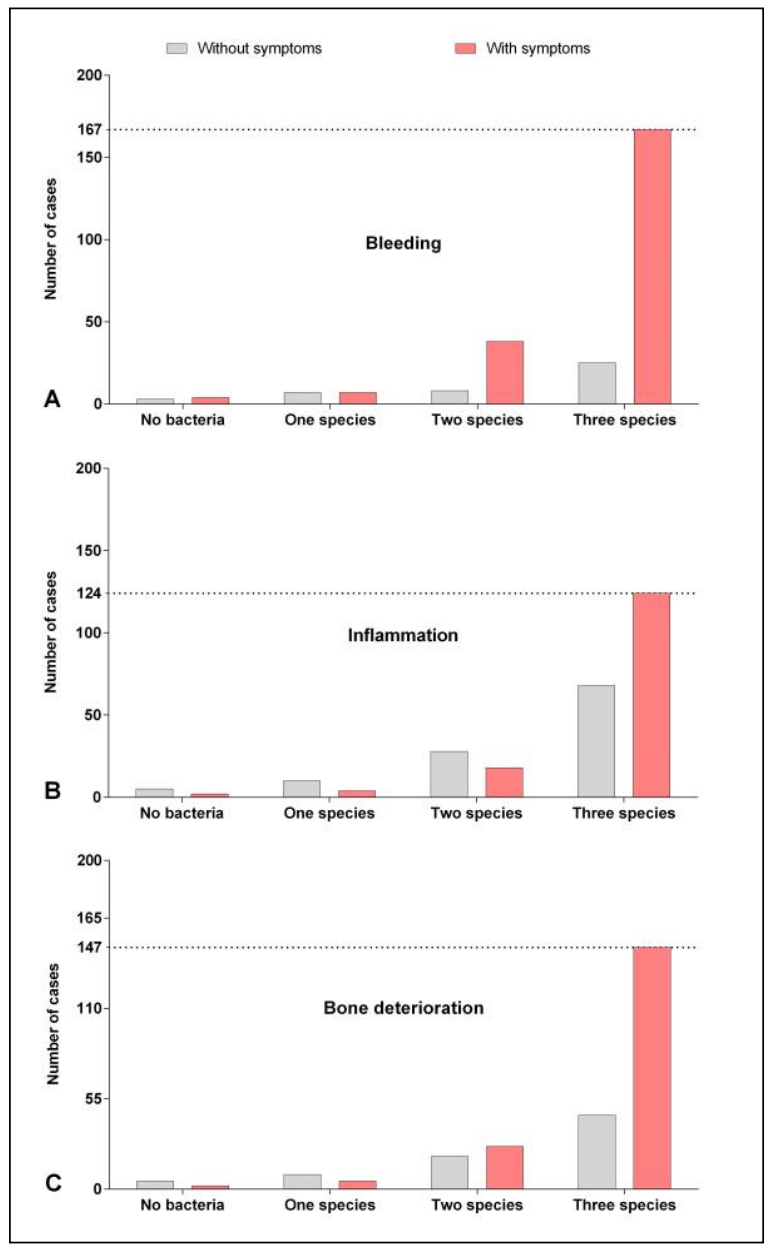

Figure 1. Associations between the number of bacterial species identified in a given patient and the presence of gingival bleeding (Figure 1A), inflammation (Figure 1B) and bone deterioration (Figure 1C) clinical symptoms. For each symptom, we emphasized the maximum number of cases presenting the symptoms, which is strongly correlated with the influences exerted concomitantly by the various bacterial species from the red complex.

A stepwise regression analysis on cases with all red complex bacteria present $(\mathrm{N}=$ 130) retained only the load of $T$. denticola as significant for the maximum probing depth $(\mathrm{t}=$ 4.47, $\mathrm{p}<10^{-3}$, R-sq adj. $=12.84 \%$, Mallows $\left.\mathrm{Cp}=2.2\right)$. When each bacterial species was individually tested (Pearson correlation), we were able to show a decrease of $A$. actinomycetemcomitans bacterial load with the maximum depth of pocket probing, although the result was not statistical significant $(\mathrm{r}=-0.241, \mathrm{p}=0.133)$. This result requires further investigation, given the facultative anaerobic nature of this species (Tanner, 2015), as well as the competitive relationship between $P$. gingivalis and A. actinomycetemcomitans. (Haraguchi et al., 2014). In contrast, red complex bacterial load appeared to be positively 
correlated with maximum probing depth, significant associations being calculated for $P$. gingivalis $(\mathrm{r}=0.238, \mathrm{p}=0.003)$ and $T$. denticola $(\mathrm{r}=0.361, \mathrm{p}<0.001)$, but not for $T$. forsythia $(\mathrm{r}=0.111, \mathrm{p}=0.161)$. A study focused on adult Brazilians demonstrated the significant prevalence of $P$. gingivalis in deep pockets, but also of $T$. forsythia (Farias et al., 2012). The fact that we identified $T$. denticola as bearing the strongest correlation with maximum probing depth could be representing a local trait, as several studies indicated that periodontal pathogens' distribution and prevalence vary with geographic location (Tettamanti et al., 2017) and ethnicity (Gatto et al., 2014). Furthermore, the latter team found that in Italian population the strongest association with pocket depth is that of T. forsythia, which supports local bacterial specificity hypothesis.
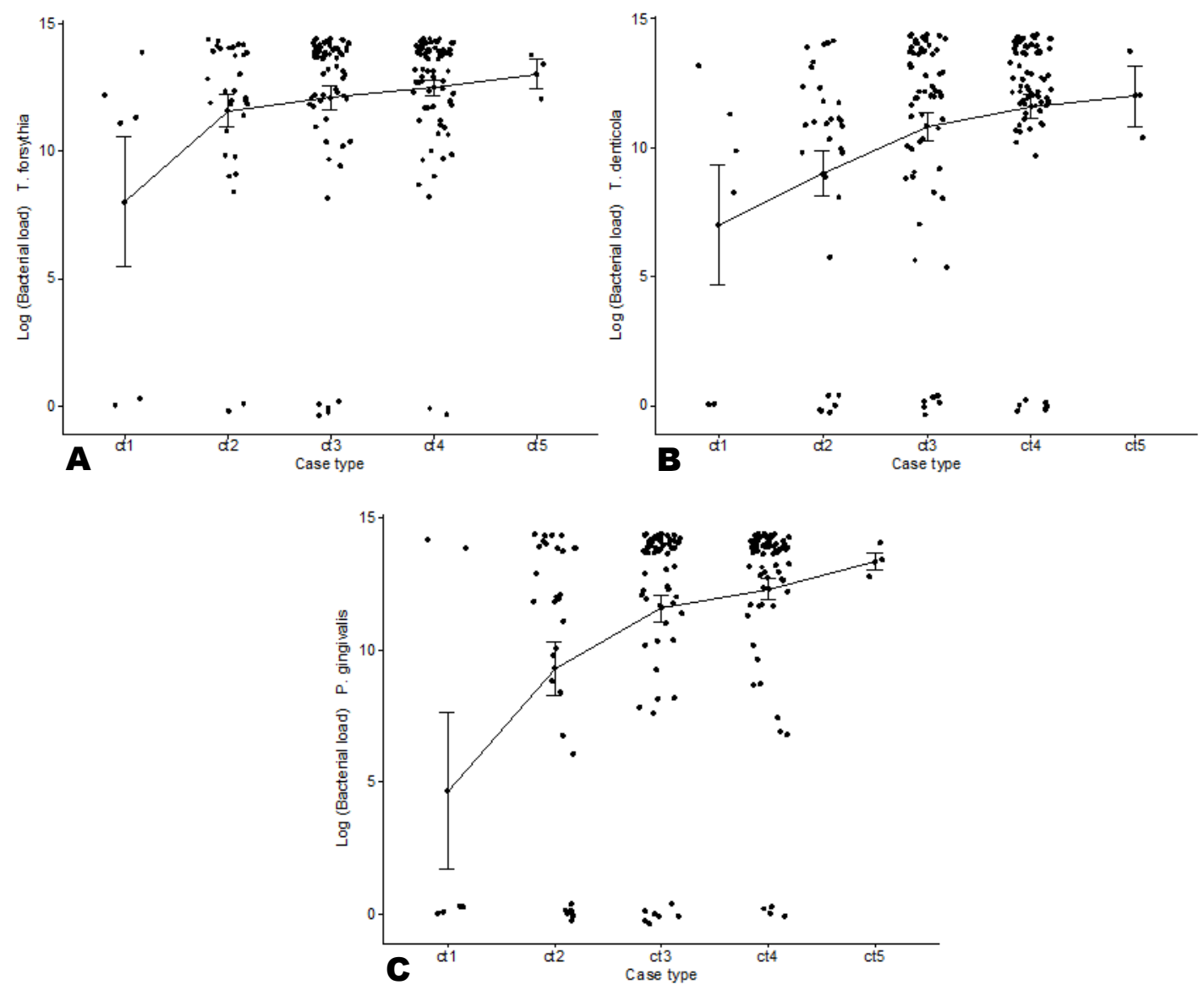

Fig. 2. Graphical representation of logarithmic distribution of bacterial load (CFUs expressed as logarithmated values) as a function of Case Type diagnosis (ct1 to ct5) for T. forsythia (A), T. denticola (B) and $P$. gingivalis (C).

A Kruskal-Wallis non-parametric ANOVA was applied to the bacterial load, separately for each red complex species, as a function of Case Type clinical diagnosis. Only 
177 subjects were classified according to this criterion. An increase was detected for all three species from Case Type 1 to Case Type 5 (figure 2), which proved significant for T. denticola $(\mathrm{H}=17.89, \mathrm{p}=0.001)$ and $P$. gingivalis $(\mathrm{H}=13.87, \mathrm{p}=0.01)$, but not for $T$. forsythia $(\mathrm{H}=$ $8.14, \mathrm{p}=0.08$ ). The Kruskal-Wallis test was chosen for its ability to control for non-normal data by analysing the ranks instead of actual bacterial load values, an aspect important for our data, which clustered in the upper part of the scale and was under-represented in the extreme Case Types 1 and 5. The results indicate an increase of the bacterial load along with the severity of diagnosis, although significant differences between levels were not always detected in a post-hoc evaluation. However, rather than strictly evaluating differences between each Case Type class, the purpose of the test was to assess the pattern of the relationship, if any. Such a tendency was also demonstrated in a Thai population (Waraaswapati et al., 2009) for the red complex bacteria. Since our analysis included patients with no bacterial load, we repeated the test without these null figures. The results remained significant only for T. denticola $(\mathrm{H}=14.68, \mathrm{p}=0.005, \mathrm{~N}=156)$, but not for $P$. gingivalis $(\mathrm{H}=$ 5.60, $\mathrm{p}=0.23, \mathrm{~N}=155)$ or $T$. forsythia $(\mathrm{H}=5.87, \mathrm{p}=0.20, \mathrm{~N}=166)$. Compared to the red complex species, A. actinomycetemcomitans bacterial load showed no trend in relationship with the dental pathology diagnosis $(\mathrm{H}=3.40, \mathrm{p}=0.33, \mathrm{~N}=43)$.

\section{Conclusion}

In our study population, coexistence of red complex bacteria is positively associated with several dental pathologies, i.e. inflammation, gingival bleeding and bone deterioration, but not with halitosis or redness. No similar association was found for $A$. actinomycetemcomitans. Bacterial load did not associate as strongly with pathology, except for $P$. gingivalis and $T$. denticola, with regard to the depth of probing and the severity of periodontal disease as clinically diagnosed (Case Type 1 to 5). P. gingivalis and T. denticola, $P$. gingivalis and $T$. forsythia, $T$. denticola and $T$. forsythia, as well as $A$. actinomycetemcomitans and T. forsythia appear to associate with each other. In contrast to red complex bacteria, A. actinomycetemcomitans was identified in relatively few cases and apparently its bacterial load decreases with the depth of dental pockets, a result that needs to be eventually validated on a larger population sample.

Both $P$. gingivalis and $T$. denticola, but especially the latter, characterize dental damage in Romanian patients. The highest prevalence of $T$. denticola in severe cases of periodontal disease as well as its strongest positive correlation with maximum pocket depth 
could be representative for the Romanian population, a hypothesis that needs to be rigorously tested.

\section{References}

1. Armitage G. C., 1995. Clinical Evaluation of Periodontal Diseases. Periodontology 20007 (February): $39-53$.

2. Armitage G.C., 2003. Diagnosis of Periodontal Diseases. Journal of Periodontology 74 (8): 1237-47.

3. Bao K., Belibasakis G.N., Thurnheer T., Aduse-Opoku J., Curtis M.A., Bostanci N., 2014. Role of Porphyromonas gingivalis gingipains in multi-species biofilm formation. BMC Microbiology 14:258.

4. Bar T., Kubista M., and Tichopad A., 2012. Validation of Kinetics Similarity in qPCR. Nucleic Acids Research 40.4 (2012): 1395-1406. PMC.

5. Bastos J. A.,. Diniz C. G,. Bastos M. G, Vilela E. M., Silva V. L, Chaoubah A., Souza-Costa D. C., and Andrade L. C. F., 2011. Identification of Periodontal Pathogens and Severity of Periodontitis in Patients with and without Chronic Kidney Disease. Archives of Oral Biology 56 (8): 804-11.

6. Chen F., Gao L., Tian B., Zhang X., Zhang Y., Dong G., Lu H. et al., 2005. Position paper: epidemiology of periodontal diseases. J periodontol 76 (2005): 1406-1419.

7. Decat E., Cosyn J., De Bruyn H., Miremadi R., Saerens B., Van Mechelen E., Vermeulen S., Vaneechoutte M., and Deschaght P., 2012. Optimization of quantitative polymerase chain reactions for detection and quantification of eight periodontal bacterial pathogens. BMC Research Notes 5 (december): 664.

8. Farias B.C., Souza P.R.E., Ferreira B., et al, 2012. Occurrence of periodontal pathogens among patients with chronic periodontitis. Brazilian Journal of Microbiology 43(3):909-916.

9. Friedrich V., Gruber C., Nimeth I., et al, 2015. Outer membrane vesicles of Tannerella forsythia: biogenesis, composition, and virulence. Molecular Oral Microbiology 30(6):451-473.

10. Gatto M. R., Montevecchi M., Paolucci M., Landini M. P., and Checchi L., 2014. Prevalence of Six Periodontal Pathogens in Subgingival Samples of Italian Patients with Chronic Periodontitis. The New Microbiologica 37 (4): 517-24.

11. Gorman M.A., Seers C.A., Michell B.J., et al., 2015. Structure of the lysine specific protease Kgp from Porphyromonas gingivalis, a target for improved oral health. Protein Science: A Publication of the Protein Society 24(1):162-166.

12. Haraguchi A., Miura M., Fujise O., et al, 2014. Porphyromonas gingivalis gingipain is involved in the detachment and aggregation of Aggregatibacter actinomycetemcomitans biofilm. Mol Oral Microbiol 29(3):131-143.

13. Kirakodu S.S., Govindaswami M., Novak M.J., Ebersole J.L., and Novak K.F., 2008. Optimizing qPCR for the Quantification of Periodontal Pathogens in a Complex Plaque Biofilm. The Open Dentistry Journal 2 (March): 49-55.

14. Lanza E., Magan-Fernandez A., Bermejo B., de Rojas J., Marfil-Alvarez R., and Mesa F., 2016. Complementary Clinical Effects of Red Complex Bacteria on Generalized Periodontitis in a Caucasian Population. Oral Diseases 22 (5): 430-37. 
15. Loyola-Rodriguez J.P., Garcia-Cortes J.O., Martinez-Martinez R.E., Patiño-Marin N., MartinezCastañon G.A., Zavala-Alonso N.V., and Amano A., 2014. Molecular Identification and Antibiotic Resistant Bacteria Isolated from Primary Dentition Infections. Australian Dental Journal 59 (4): 497503.

16. Mariotti A. and Arthur F. H., 2015. Defining Periodontal Health. BMC Oral Health 15 (Suppl 1): S6.

17. Masunaga H., Tsutae W., Oh H., Shinozuka N., Kishimoto N., and Ogata Y., 2010. Use of Quantitative PCR to Evaluate Methods of Bacteria Sampling in Periodontal Patients. Journal of Oral Science 52 (4): $615-21$.

18. Peirson S. N., Butler J. N., and Foster R. G.,2003. Experimental Validation of Novel and Conventional Approaches to Quantitative Real-Time PCR Data Analysis. Nucleic Acids Research 31.14 (2003): e73.

19. Socransky S. S., Haffajee A. D., Cugini M. A., Smith C., and Kent R. L., 1998. Microbial Complexes in Subgingival Plaque. Journal of Clinical Periodontology 25 (2): 134-44.

20. Suzuki N., Yoneda M., Hirofuji T., 2013. Mixed Red-Complex Bacterial Infection in Periodontitis. International Journal of Dentistry 2013:587279.

21. Tanner A.C.R., 2015. Anaerobic culture to detect periodontal and caries pathogens. Journal of Oral Biosciences 57(1), 18-26.

22. Teles R., Teles F., Frias-Lopez J., Paster B., and Haffajee A., 2013. Lessons learned and unlearned in periodontal microbiology. Periodontology 200062 (1): 95-162.

23. Tettamanti L., Gaudio R. M., Cura F., Mucchi D., Illuzzi N., and Tagliabue A., 2017. Prevalence of Periodontal Pathogens among Italian Patients with Chronic Periodontitis: A Retrospective Study on 2992 Patients. ORAL \& Implantology 10 (1): 28-36.

24. The Research, Science and Therapy Comitee of the American Academy of Periodontology, 1999. Informational paper: The Pathogenesis of Periodontal Diseases. Journal of Periodontology 70 (4): 457 70 .

25. Wara-aswapati N., Pitiphat W., Chanchaimongkon L., Taweechaisupapong S., Boch J. A., and Ishikawa I., 2009. Red Bacterial Complex Is Associated with the Severity of Chronic Periodontitis in a Thai Population. Oral Diseases 15 (5): 354-59. 